\title{
ANÁLISIS Y COMPARATIVO DE LOS AMPLIFICADORES ÓPTICOS SOA, EDFA Y RAMAN
}

\section{Análisis y comparativo de los amplificadores ópticos soa, edfa y raman}

\author{
Sergio Moreno ${ }^{1}$, Daniel Torres ${ }^{1}$, Gustavo Puerto ${ }^{2}$ \\ ${ }^{1}$ Esp. Universidad Pedagógica y Tecnológica de Colombia (Tunja, Colombia). sergioalfredo.moreno@uptc.edu.co, \\ wilmer.torres@uptc.edu.co. \\ 2PhD. Universidad Distrital Francisco José de Caldas (Bogotá-Colombia).gapuerto@udistrital.edu.co
}

(Recibido septiembre 21 de 2017 y aceptado noviembre 08 de 2017)

\section{Resumen}

En este documento, se expone de manera general la estructura y el funcionamiento de los amplificadores ópticos SOA, EDFA y Raman, además, se presenta una comparación entre las tecnologías ópticas mencionadas anteriormente, en donde se logran identificar los campos de acción de cada una de ellas, y las ventajas de los modelos híbridos de amplificación óptica. Asimismo, se abordará el tema de las no linealidades y su contribución.

Palabras clave: Amplificación; atenuación; fibra óptica; fotónica; óptica.

\section{Abstract}

En este documento, se expone de manera general la estructura y el funcionamiento de los amplificadores ópticos SOA, EDFA y Raman, además, se presenta una comparación entre las tecnologías ópticas mencionadas anteriormente, en donde se logran identificar los campos de acción de cada una de ellas, y las ventajas de los modelos híbridos de amplificación óptica. Asimismo, se abordará el tema de las no linealidades y su contribución.

Keywords: Amplificación; atenuación; fibra óptica; fotónica; óptica.

\section{INTRODUCCIÓN}

Las telecomunicaciones han tenido una gran evolución y crecimiento en las últimas décadas, en los últimos años se ha visto un aumento incesante en la transmisión de información en los sistemas de comunicaciones, que han forzado la creación de mecanismos capaces de soportar y gestionar estos grandes volúmenes de datos. Con el paso del tiempo, la transferencia de información se convirtió en un factor crítico, surgió la necesidad de un medio guiado que tuviera la capacidad de soportar el crecimiento exponencial del flujo de información. Por lo anterior, se optó por la tecnología óptica, la cual permite el envío de señales fotónicas a una velocidad mucho mayor que con cualquier otro sistema, brinda seguridad en la transmisión de datos, posee inmunidad a interferencias electromagnéticas, entre otras características. En este sentido, la fotónica se ha posicionado como la tecnología dominante, pues permite explotar el ancho de banda de la fibra óptica, a tal punto que en la actualidad se habla de redes y sistemas todo-ópticos [1][2].

A pesar que un sistema de comunicaciones óptico presenta una serie de ventajas, después de que una señal ha viajado una distancia considerable, ésta se atenúa debido a las

pérdidas de potencia a lo largo de la fibra, a imperfecciones de la misma en el proceso de fabricación, o a curvaturas, en

ese punto dicha señal requiere un incremento de en el nivel de potencia. Tradicionalmente, la señal óptica era convertida a una señal eléctrica, amplificada eléctricamente y convertida de regreso en una señal óptica. Por el contrario, un amplificador óptico permite incremen- 
tar el nivel de potencia de la señal en el dominio óptico, sin necesidad de pasar la señal al dominio eléctrico (conversión opto-electrónica) [3]. El amplificador óptico proporciona una solución más simple al problema de la atenuación y puede ser usado independientemente del tipo de modulación y del ancho de banda, además es un dispositivo bidireccional y permite el trabajo en sistemas con multiplexación por división de longitud de onda [4][5].

La invención del láser de semiconductor en la década de 1960, permitió realizar los primeros estudios del amplificador óptico de semiconductor, comúnmente conocido como SOA. Su desarrollo se centró en un dispositivo de amplificación óptica que se desempeñaba alrededor de los $820 \eta n$, pues en esa década no había láseres ni receptores ópticos que operaran en segunda y tercera ventana. A pesar de esto, a mediados de los 80 se dieron los primeros avances de un SOA que operaba en las regiones de 1300 nm y 1550 nm [2]. Fue entonces, cuando en la Universidad de Southampton en el año de 1985, se acuñó el concepto de fibras ópticas dopadas con iones de tierras raras ( $\mathrm{Nd}, \mathrm{Er}, \mathrm{Pr}, \mathrm{Ho}, \mathrm{Tm}$ ), dando inicio a una nueva generación de fibras, las cuales serían usadas como un novedoso modelo de amplificador óptico. El EDFA (amplificador óptico de fibra dopada con erbio), presentó un interés especial debido a que el ion Er3+ posee una transición alrededor de los $1.536 \mu \mathrm{m}$, lo cual permite la amplificación de las señales en los sistemas de comunicaciones ópticas modernos [6].

Las distintas tecnologías emergentes de amplificación óptica propulsaron una serie de avances importantes en este campo, pocos años después del surgimiento del EDFA, se logró la transmisión de pulsos ópticos que conservaban su forma al propagarse a lo largo de una fibra óptica sin presentar pérdidas, contrarrestando el efecto de la dispersión por medio de no linealidades. En 1988 un experimento de laboratorio consiguió la transmisión de datos a una distancia de aproximadamente $4000 \mathrm{~km}$, compensando las pérdidas de la fibra mediante la dispersión estimulada de Raman. Desde entonces, se han aprovechado efectos no lineales en la fibra óptica para el desarrollo de tecnologías laser y de amplificación Raman [7].

Este articulo presenta un paralelo entre las tres tecnologías de amplificación óptica mencionadas anteriormente, en donde se señalan sus características principales, ventajas y desventajas, permitiendo al lector identificar los campos de acción asociados a cada una de ellas, adicionalmente se pretende indicar la viabilidad de la combinación de dos tecnologías fotónicas de amplificación, con el fin de establecer si los modelos ópticos híbridos presentan algún tipo de ventaja respecto a los modelos ópticos convencionales, se encuentra que ningún modelo de amplificación es ideal, pero ciertas características específicas permiten posicionar determinada tecnología de amplificación óptica como la dominante en los sistemas de comunicación óptica modernos, cabe resaltar que el estudio realizado destaca que las no linealidades pueden contribuir en un sistema óptico, sin limitarse a la amplificación.

\section{AMPLIFICADORES ÓPTICOS SOA, EDFA Y RAMAN}

"Se le considera un amplificador óptico, cuando la señal no necesita ser convertida a una señal eléctrica, amplificarla y volverla óptica nuevamente" [8].

\subsection{Amplificador SOA}

Un SOA es un dispositivo opto-electrónico que genera un aumento en la amplitud de la señal fotónica que se propaga a través de él, dicha señal pasa de la fibra óptica al amplificador, es amplificada y finalmente regresa a la fibra. Para que esto suceda es necesario que el dispositivo se encuentre en región activa, además de estar polarizado de forma correcta de acuerdo a una corriente eléctrica. En la figura 1 se observa el diagrama esquemático de un SOA[2].

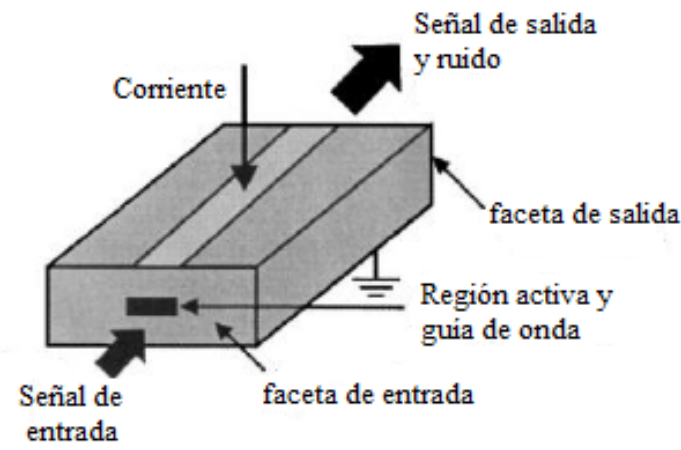

Figura 1. Esquema del amplificador SOA [2].

Una de las desventajas de los amplificadores ópticos de semiconductor es el nivel de ruido que posee la señal fotónica a la salida del dispositivo, este factor es causado por el propio SOA y no se puede eliminar completamente [2]. 
Los SOA se dividen en dos arquitecturas, amplificador óptico de semiconductor de caras reflectantes (Fabry Perot o FP-SOA) y amplificador óptico de semiconductor de onda viajera (OV-SOA). La diferencia radica en que el primero tiene reflexiones muy significativas y variantes en sus caras. Gracias a sus revestimientos anti-reflectivos, la reflexión en el OV-SOA es despreciable. Además, el OV-SOA no es tan sensible a las variaciones en la corriente de polarización, polarización de la señal y temperatura de trabajo. En la figura 2, se expone la estructura de los amplificadores mencionados anteriormente y el comportamiento de la ganancia para cada caso, de acuerdo a la longitud de onda [2].

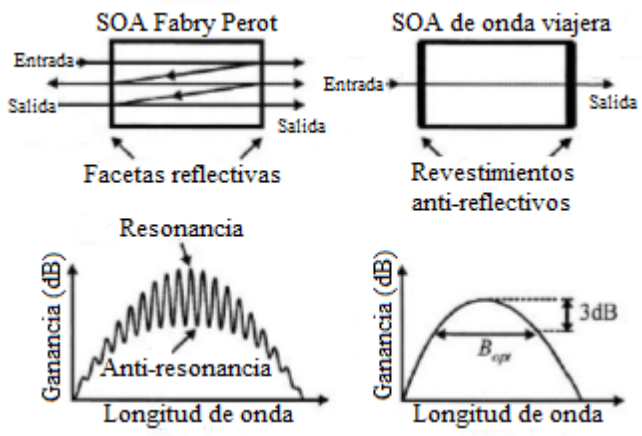

Figura 2. Comparación de los FP-SOA y TW-SOA, Diagrama de estructura respecto a la señal y espectros de ganancia [2].

En un amplificador óptico de semiconductor, una fuente de corriente externa es la encargada de inyectar electrones con el fin de generar un mecanismo de emisión estimulada, el cual es el principio de funcionamiento del SOA para la amplificación. Al incidir un fotón en el semiconductor ocurre lo que se denomina recombinación estimulada, como consecuencia, un electrón pasa de la banda de conducción a la banda de valencia, generando un nuevo fotón con la misma frecuencia y fase del fotón que inicio el proceso, al ocurrir esto se tienen dos fotones idénticos, pues el primer fotón no se extingue, a este proceso se le conoce como emisión estimulada [2].

\subsection{Amplificador EDFA}

El amplificador óptico de fibra dopada con erbio (EDFA=Erbium Doped Fiber Amplifier) ha generado especial interés para las comunicaciones ópticas debido a que el ion $\mathrm{Er} 3+$ posee una transición láser alrededor de $1.536 \eta \mathrm{m}$, lo cual permite amplificar las señales de los sistemas de comunicaciones por fibra óptica en la terce- ra ventana (longitudes de onda entre $1.53 \eta \mathrm{m}$ y $1.55 \mathrm{~nm}$ ) [6].

En la figura 3, se muestra la configuración básica de un amplificador óptico EDFA; en este dispositivo se puede observar como por medio de un acoplador la fibra dopada queda de forma transversal a la fuente de bombeo, de esta manera una señal que presenta atenuación es amplificada cuando pasa por la fibra dopada con iones de Erbio (Er). El acoplador utilizado en este dispositivo logra que la radiación de bombeo pase a la línea de transmisión y evita que la radiación de la señal que se amplifica pase a la rama del bombeo [6].

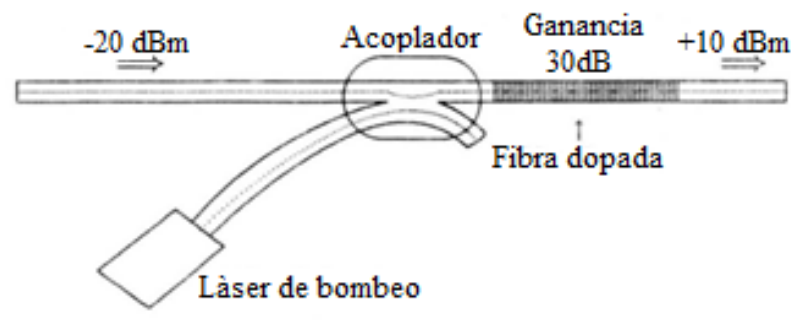

Figura 3. Configuración básica del amplificador óptico de fibra dopad [6].

El Er tiene una característica física que le permite reaccionar a los fotones emitidos por un haz de luz, dicha característica permite acelerar la producción de fotones lo cual representa en un sistema de transmisión óptico un aumento significativo en la potencia óptica [8].

El Er puede reaccionar a una longitud de onda de 1480nm o 980nm, por lo tanto, el funcionamiento de un EDFA depende de una potencia de bombeo que permita reaccionar a los iones de Er. En la figura 4, se muestra un esquema de bombeo en el que se ingresa la señal a amplificar, y la señal de bombeo (1480 o 980nm) combinadas por un multiplexor al EDFA, lo anterior provoca que las partículas de Er reaccionen a los fotones de luz, con la producción de más fotones se incrementa la potencia de las dos señales introducidas. Al final del sistema se utiliza un filtrado óptico que permite separar la señal amplificada de la señal de bombeo, permitiendo el paso únicamente de la señal amplificada [8]. 


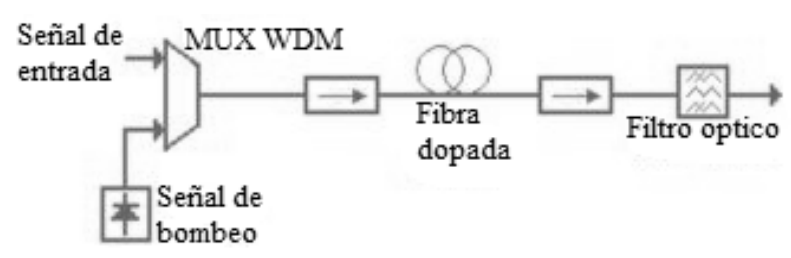

Figura 4. Esquema general del amplificador óptico de fibra dopada [8].

\subsection{Amplificador RAMAN}

Los amplificadores Raman se valen de la ganancia Raman de la propia fibra óptica. Dicha ganancia responde a un efecto no lineal producido en la molécula de $\mathrm{SiO} 2$ (Dióxido de Silicio) que compone la fibra, y que dada la disposición de esta como guía de onda se estimula en la propia propagación. La Figura 5 muestra los espectros de ganancia Raman para diferentes tipos de fibra óptica. Como se puede ver en dicha figura, la ganancia Raman produce un espectro de ganancia de aproximadamente $20 \mathrm{THz}$ a partir de la longitud de onda de bombeo, esta ganancia se produce en cualquier longitud de onda, con un máximo de $13 \mathrm{THz}$ de la frecuencia de bombeo (aproximadamente la ganancia Raman tiene una anchura de 150 nm por encima de la longitud de onda de bombeo en la ventana de 1550 nm y el máximo de ganancia se produce en esta ventana a $100 \mathrm{~nm}$ del bombeo). El problema de los amplificadores Raman es que requieren de grandes potencias de bombeo (>0.5 W) [9].

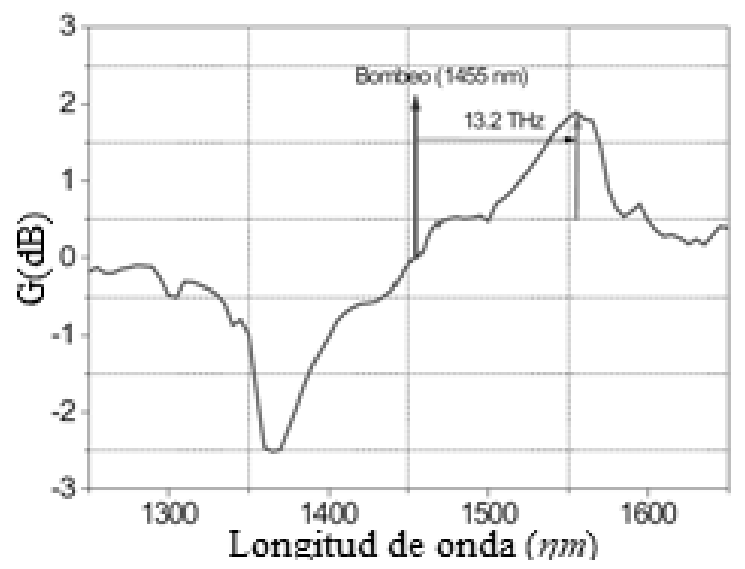

Figura 5. Espectro de ganancia Raman para diferentes tipos de fibra óptica [10].

\section{ANALISIS Y COMPARACIÓN}

En [1] se afirma que a pesar que las no linealidades del SOA contribuyeron a que esta tecnología fuera reemplazada por los EDFA, fue precisamente esta característica la que permitió a los amplificadores ópticos de semiconductor centrarse en aplicaciones de procesado como la conmutación todo óptica, la conversión de longitud de onda en sistemas WDM, realizar compuertas lógicas ópticas, comportarse como un generador de pulsos y generar filtros dinámicamente configurables. No obstante, en la actualidad se cuenta con sistemas ópticos locales que hacen uso de los SOA como dispositivo de amplificación óptica.

Los amplificadores ópticos SOA poseen las ventajas del control por corriente y un manejo de ancho de banda considerable, además son compactos e integrables, pero su sensibilidad de polarización, diafonía, factor de ruido alto y potencia de saturación pequeña lo convierten en una alternativa de amplificación óptica no muy buena.

El uso del amplificador de guía de onda quedó relegado a un segundo plano al desarrollarse los EDFA (amplificadores de fibra dopada con iones de Er) ya que estos últimos presentan un comportamiento lineal y unas constantes de recombinación del orden de ms ( $\eta$ para los SOAs). Esto permite que sean utilizados en enlaces WDM, ya que es posible la amplificación sin crosstalk entre los canales. Los EDFA son utilizados en mayor parte para comunicaciones a gran distancia, por lo cual son de gran vitalidad en cables submarinos gracias a su aplicabilidad y grandes prestaciones. Además, presentan otras ventajas, como las bajas pérdidas de acoplo a la fibra óptica, reflexiones despreciables, independencia a la polarización, alta ganancia, alta potencia de saturación de salida y baja figura de ruido ( $N F$, Noise Figure). Como contrapartida son amplificadores caros (necesitan bombeo óptico) y no trabajan en segunda ventana [1].

Los amplificadores Raman se utilizan en configuraciones de contra-propagación, la mayor parte de la amplificación se concentra al final del trayecto, por lo cual evitan superar el umbral de efectos no lineales, la amplificación Raman reduce la emisión de ruido de los EDFA lo cual es ideal para los sistemas de amplificación híbridos EDFA/RAMAN, como desventaja vemos que requiere de una considerable potencia de bombeo para lograr prestaciones aceptables. En la siguiente tabla [11], se

Rev. Ing. Investig. Desarro. Vol.18 (2). pp. 22-28, Julio-Diciembre. 2018, Sogamoso-Boyacá, Colombia. 
exponen las principales características de los amplificadores SOA y EDFA.

Tabla 2. Características principales de los amplificadores SOA y EDFA

\begin{tabular}{ccc}
\hline Característica & SOA & EDFA \\
\hline $\begin{array}{c}\text { Ganancia }(\mathrm{dB}) \\
\begin{array}{c}\text { Potencia de saturación } \\
(\mathrm{dBm})\end{array}\end{array}$ & $10-15$ & 30 o mas \\
\hline Factor de ruido $(\mathrm{dB})$ & $5-6$ & Mínimo 17 \\
\hline Dimensiones $(\mathrm{m})$ & Menores a & $10-100$ \\
\hline Banda de paso $(\mathrm{nm})$ & 0.001 & $20-30$ \\
\hline Margen $(\mathrm{nm})$ & $30-60$ & $1530-$ \\
& $1300-1550$ & 1565 \\
\hline Integración & Posible & Imposible \\
\hline Anchura de banda de $\lambda(\mathrm{nm})$ & 40 & 35 \\
\hline
\end{tabular}

De la tabla anterior se resalta que el SOA presenta un mejor factor en cuanto a dimensiones, banda de paso, margen, integración, y ancho de banda. En los EDFA las características dominantes respecto a los SOA son la ganancia, potencia de saturación y factor de ruido. Haciendo un balance entre las ventajas de cada tecnología de amplificación, se evidencia que las características más significativas juegan a favor de los EDFA, haciendo más atractiva esta tecnología de amplificación. En la figura 6 se exponen las respuestas de los amplificadores de onda viajera OV-SOA, el amplificador de fibra dopada con Ery el amplificador de fibra de efecto Raman. Se resalta que las tres tecnologías de amplificación poseen anchos de banda similares, aunque en el SOA dicho factor es ligeramente mayor, además, es claro que los amplificadores ópticos EDFA y Raman poseen una ganancia superior, comparada con la del SOA.

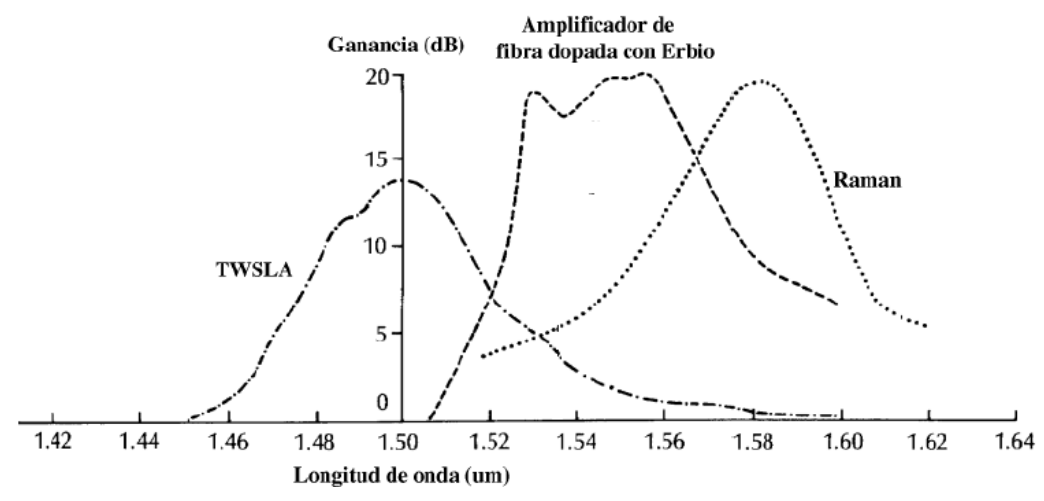

Figura 6. Características de amplificadores ópticos para la banda de 1.5 $\mu \mathrm{m}$ [4].

El amplificador de efecto no lineal (Raman) es el competidor del EDFA en la banda de los $1500 \mathrm{~nm}$, pero al realizar la comparación entre los dos sistemas ópticos, el amplificador predilecto es el de fibra dopada, pues además de las ventajas mencionadas en los párrafos anteriores, se destaca su versatilidad y costo reducido. En la siguiente tabla se expone la comparación entre las tecnologías de amplificación óptica EDFA y Raman [12].

Tabla 3. Características principales de los amplificadores EDFA y Raman

\begin{tabular}{|c|c|c|}
\hline Característica & EDFA & Raman \\
\hline Amplificación de banda & $\begin{array}{l}\text { Las bandas "C" y "L" (alrededor } \\
\text { de } 1500 \\
\eta m \text { ); no trabajan en el rango de } \\
1300 \eta \mathrm{m} \text {. }\end{array}$ & $\begin{array}{l}\text { Depende de la disponibilidad } \\
\text { de bombas de longitud de } \\
\text { onda; funciona en todas las } \\
\text { bandas. }\end{array}$ \\
\hline Ganancia & $\begin{array}{l}30 \text { dB o más dependiendo de la } \\
\text { concentración de los iones, la } \\
\text { longitud de la fibra y la configura- } \\
\text { ción de las bombas. }\end{array}$ & $\begin{array}{l}\text { De } 4 \text { a } 11 \mathrm{~dB} \text {, proporcional a } \\
\text { la intensidad de la bomba y la } \\
\text { efectividad de la longitud de la } \\
\text { fibra }\end{array}$ \\
\hline
\end{tabular}


Análisis y comparativo de los amplificadores ópticos soa, edfa y raman

\begin{tabular}{lll}
\hline \multicolumn{1}{c}{ Característica } & \multicolumn{1}{c}{ EDFA } & \multicolumn{1}{c}{ Raman } \\
\hline Potencia de saturación & $\begin{array}{l}\text { Depende de la ganancia y las con- } \\
\text { stantes del material. }\end{array}$ & $\begin{array}{l}\text { Se aproxima a la potencia de } \\
\text { las ondas de la bomba. }\end{array}$ \\
\hline Ruido & Amplifica el Ruido. & No amplifica el ruido. \\
\hline $\begin{array}{l}\text { Longitud de onda de la } \\
\text { bomba }\end{array}$ & $980 \eta \mathrm{nm}$ o $1480 \mathrm{~nm}$. & $\begin{array}{l}\text { Aproximadamente } 100 \mathrm{~nm} \text { de- } \\
\text { bajo de la longitud de onda de } \\
\text { la señal en ganancia pico. }\end{array}$ \\
\hline Espaciamiento & 80 a $100 \mathrm{~km}$. & Alrededor de $65 \mathrm{~km}$. \\
\hline
\end{tabular}

Los sistemas de comunicaciones ópticos modernos buscan integrar las tecnologías ópticas de amplificación EDFA y Raman. Los amplificadores híbridos EDFA/Raman se han convertido en una ciencia emergente muy favorable, pues permiten la maximización del segmento de amplificación, como se expone en la figura 7, obteniendo como resultado una minimización de los efectos de no linealidades en la fibra, además mejorar el ancho de banda de los amplificadores de fibra dopada con $\mathrm{Er}$ [13].

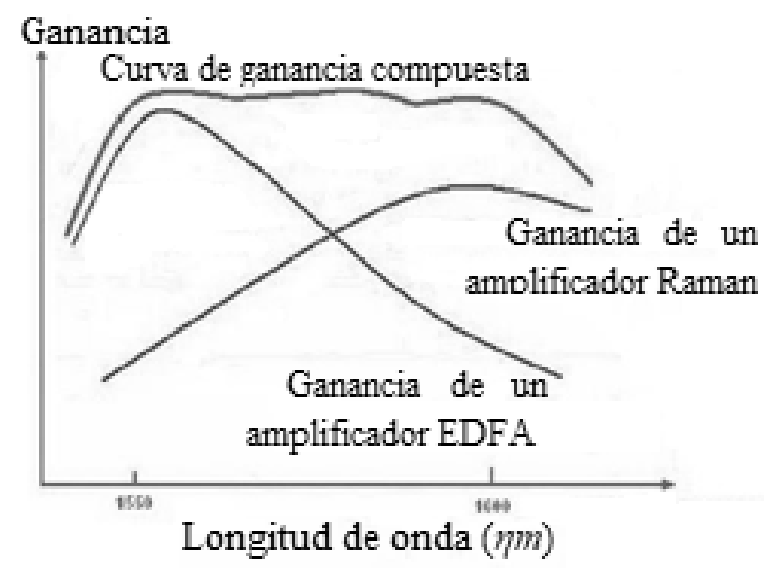

Figura 7. Ganancia compuesta de un EDFA y un Raman [10].

\section{CONCLUSIONES}

Las tres principales clases de amplificadores ópticos que existen actualmente son los EDFA, Raman y SOA, En este artículo se logra determinar que el amplificador dominante en los sistemas de comunicación ópticos es el EDFA, pues presenta unas ventajas significativas como el ancho de banda, potencia de salida y características de ruido mínimas, además proporciona ventajas funcionales y de coste significativas que se ven reflejadas en mejores prestaciones en función de la potencia de transmisión óptica. Ideal en aplicaciones para comunicaciones a gran distancia como redes de área amplia y comunicaciones inter continentales.

\section{REFERENCIAS}

[1] M. D. Manzanedo Martínez (Universidad politecnica de V), "Aplicación de los amplificadores ópticos de semiconductor a la fotónica de microondas", 2013.

[2] V. M. Perilla (Universidad distrital F. J. de C), “Análisis del amplificador óptico de semiconductor y su aplicación a sistemas de regeneración de onda 2R", 2015.

[3] M. Castro (Universidad N. A. de M), “Ampliación del ancho de banda en una red de fibra óptica empleando un sistema de multiplexación por divisiones en longitudes de onda densas (dwdm)", 2014.

[4] “Amplificación óptica y óptica integrada”, pp. 8290.

[5] J.S. Castellanos-Casas, C.S. Rojas-Piña, \& G.A. Puerto-Leguizamón,"Diseño de una red óptica pasiva bidireccional con particionamiento espectral de una fuente de banda ancha", Rev. Ingeniería, Investigación y Desarrollo, Vol. 18 [1], pp. 25-33, 2018. DOI: https://doi.org/10.19053/1900771X. v18.n1.2018.7852

[6] P. Torres, "Análisis por Elementos Finitos del Amplificador de Fibra Optica Dopada con Erbio", 1995.

[7] C. Jaen (Universidad P. de M), "Estudio de limitaciones en sistemas WDM", 2002.

[8] J. H. Hinojosa Erazo (Escuela P. N. de E), "Diseño de una propuesta de red de acceso para brindar servicios de internet, telefonía, datos y televisión a través de FTTH, para el sector de Iñaquito mediante la tecnología RFOG para un proveedor de CATV", 2014.

[9] M.Gutiérrez Hernández (Universidad P. de M), “Diseño, construcción y puesta a punto de un circuito de alimentación de láseres de diodo de alta potencia y amplificadores ópticos de semiconductor", 2005. 
[10] S. Martín López, (Universidad C. de M) Generacion de supercontinuo en fibras opticas monomodo con fuentes de bombeo continuo. 2007.

[11] S. Y. R. Digitales, "Definición y métodos de prueba de los parámetros genéricos pertinentes de los dispositivos y subsistemas de amplificadores ópticos", 2006.
[12] M. Magaña Loyo (Instituto P. N. de M), “Amplificador universal Raman para equipo OTDR en enlaces ópticos de larga distancia", 2015.

[13] A. O. Hernández Calvario, "Diseño, caracterización e implementación de un amplificador versátil de fibra dopada con erbio", 2010. 